\title{
COMPUTATIONAL PREDICTION OF THE STABILITY OF TENSEGRITY STRUCTURES
}

\author{
Z. Bieniek ${ }^{1}$, I. Mascolo ${ }^{2}$, A. Amendola ${ }^{2}$, A. Micheletti ${ }^{3}$, R. Luciano ${ }^{4}$ and F. Fraternali ${ }^{2}$ \\ ${ }^{1}$ Faculty of Civil and Environmental Engineering and Architecture, \\ Rzeszów University of Technology, Rzeszów, Poland \\ e-mail: zbieniek@prz.edu.pl \\ ${ }^{2}$ Department of Civil Engineering, University of Salerno \\ e-mail: \{i.mascolo,adaamendola1, f.fraternali\}@unisa.it \\ ${ }^{3}$ Department of Civil Engineering and Computer Science, University of Rome Tor Vergata, Italy \\ e-mail: micheletti@ing.uniroma2.it \\ ${ }^{4}$ Department of Engineering, University of Naples “Parthenope”, Naples (Italy), email: \\ raimondo.luciano@uniparthenope.it
}

\begin{abstract}
The lightweight nature of tensegrity structures calls for the formulation of computational tools that are able to analyze the stability problem of such structures, both in statics and dynamics. The present work analyzes the concepts of prestress-stability and superstability with reference to a special class of tensegrity structures recently appeared in the literature, named class theta tensegrity prisms. Such structures are formed by two triangular prisms superimposed one over the other, and two different sets of strings connecting the two prisms. The given results can be easily generalized to general tensegrity structures and can be usefully incorporated in formfinding and topology optimization codes.
\end{abstract}

Keywords: class $\theta$ tensegrity systems, form-finding, prestress-stability, superstability. 


\section{INTRODUCTION}

Tensegrity structures are three dimensional mechanical systems formed by a discontinuous set of compressive elements (bars or struts) connected each other through tensile elements (cables or strings). Such systems differ from conventional truss structure due to the fact that the equilibrium of the structure is guaranteed by the action of self-equilibrated sets of axial forces in bars and cables (in absence of external loads), which stabilize infinitesimal mechanisms from the freestanding configuration. Structural problems of relevant importance to tensegrity systems are related to the existence of freestanding configurations, and the stability of the static and dynamic equilibrium problems under the action of external forces [1]-[7]. It is worth remarking that tensegrity structures offer lightweight solutions to structural design problems under a large variety of loading conditions [8]-[15].

A new class of tensegrity structures has been recently introduced in the literature, with the name of class theta tensegrity prisms [16]. Such structures are formed by the superimposition of two T3 prisms (i.e., two triangular prisms formed by 3 bars each [Skelton and de Oliveira]), which are connected each other through two different set of cables. A first set of cables connects the external nodes of the structure, while the second set connects the inner nodes and provides a connection between the two superimposed prisms. The Greek letter has been introduces by Bieniek to label such systems [16], since it mimics the geometry of a structure formed by external and internal cables.

Due to the presence of self-stress and infinitesimal mechanisms, different notions of stability can be introduced with reference to tensegrity systems [17], [18], [19], with special emphasis on prestress-stability and superstability (Sect. 2). The present work specializes such definitions with reference to class theta tensegrity prisms (Sect. 3). A benchmark example referred to this structural typology is analyzed through a numerical approach (Sect. 4), which can be easily generalized to study the stability of arbitrarily shaped tensegrity systems.

\section{STABILITY NOTIONS FOR TENSEGRITY STRUCTURES}

Let us consider the current configuration of an elastic tensegrity structure formed by a total number $M$ of bars and strings that obey the following constitutive law

$$
t_{m}=k_{m}\left(\ell_{m}-\bar{\ell}_{m}\right), m=1, \ldots, M
$$

where $\bar{\ell}_{m}$ denotes the length of the generic member in the unstressed configuration of the lattice (rest-length) and $k_{m}=E_{m} A_{m} / \bar{\ell}_{m}$ denotes the axial stiffness coefficient. Here, the quantity $E_{m}$ denotes the Young modulus of the $m$-th member, while $A_{m}$ denotes the cross-section area.

The equilibrium problem of the lattice is written as follows [19]:

$$
\boldsymbol{A} \boldsymbol{t}=\boldsymbol{f}
$$

where $\boldsymbol{A}$ denotes the instantaneous static matrix of the structure; $\boldsymbol{t} \in \mathbb{R}^{M}$ denotes the vector of the axial forces carried by bars and strings; and $f \in \mathbb{R}^{Q}$ denotes the external force vector. $Q$ being the total number of degrees of freedom of the structure. The transpose $\mathbf{B}$ of the static matrix rules the kinematic problem of the structures, and its null space groups the infinitesimal mechanisms of the structure [19]. The equilibrium problem of the structure is ruled by the equations: 


$$
\sum_{m=1}^{M} k_{m}\left(\ell_{m}-\bar{\ell}_{m}\right) \frac{\partial \ell_{m}}{\partial q_{r}}=f_{r},
$$

where $\partial \ell_{m} / \partial q_{r}$ is the $(m, r)$ entry of $\boldsymbol{A}$. By differentiating both sides of Eqn. (3) one time with respect to time, and denoting time-derivatives by superimposed dots, we obtain the incremental form of the equilibrium problem as follows

$$
K_{T} \dot{\boldsymbol{q}}=\dot{\boldsymbol{f}} .
$$

Here, $\boldsymbol{K}_{\boldsymbol{T}}$ is the tangent stiffness matrix of the structure, which is computed through the summation of the material stiffness matrix $\boldsymbol{K}_{M}$ and the geometric stiffness matrix $\boldsymbol{K}_{G}$.

In particular, $\boldsymbol{K}_{M}$ depends on the material stiffness coefficients $\left(k_{1}, \ldots, k_{m}\right)$ and on the cosine directors of the member axes $\left(\frac{\partial \ell_{m}}{\partial q_{r}}\right)$. It can be written as follows:

$$
K_{M_{r s}}=k_{m} \frac{\partial \ell_{m}}{\partial q_{r}} \frac{\partial \ell_{m}}{\partial q_{r}}, \quad(r, s=1, \ldots, Q),
$$

Moreover, $\boldsymbol{K}_{G}$ depends on the tensions acting in the current configurations of the members $\left(t_{1}, t_{2}, \ldots, t_{M}\right)$ and on the variations of the cosine directors of the member axes (i.e., $\left.\frac{\partial^{2} \ell_{m}}{\partial q_{r} \partial q_{s}}\right)$ :

$$
K_{G_{r s}}=t_{m} \frac{\partial^{2} \ell_{m}}{\partial q_{r} \partial q_{s}}, \quad(r, s=1, \ldots, Q) .
$$

From Eqn. (6), it results that the geometric term of $\boldsymbol{K}_{\boldsymbol{T}}$ is null when the members of the structure are not in tension or in case of infinitesimally-small deformations.

By analyzing Eqn. (5), we can deduce that $\boldsymbol{K}_{M}$ can be also written in the following form:

$$
\boldsymbol{K}_{M}=\boldsymbol{A} \operatorname{diag}\left(k_{1}, k_{2}, \ldots, k_{M}\right) \boldsymbol{A}^{T} .
$$

This means that it is reasonable to write that $\boldsymbol{B}=\mathbf{A}^{\boldsymbol{T}}$ ([18][20][21]).

Then, the expression $\boldsymbol{K}_{\boldsymbol{M}} \boldsymbol{u}_{\boldsymbol{M}}=\mathbf{0}$ representing a zero-material-stiffness mode, has the same meaning of writing $\dot{\boldsymbol{q}}_{M}$ multiplied by an infinitesimal time interval (that represents the mechanism of the structure), where the quantity $\boldsymbol{u}_{M}$ is the virtual displacement.

If $\boldsymbol{K}_{\boldsymbol{T}}$ is positive-definite in correspondence with a generic configuration, the latter is defined as stable and it results

$$
\boldsymbol{K}_{T} \boldsymbol{u} \cdot \boldsymbol{u}>0
$$

where $\boldsymbol{u}$ is a generic virtual displacement from the current configuration [17][18][21][22]. Moreover, we can define a prestress-stable configuration if it results

$$
\boldsymbol{K}_{G} \boldsymbol{u}_{M} \cdot \boldsymbol{u}_{M}>0
$$

in correspondence with each non-trivial mechanism $\left(\boldsymbol{u}_{M} \neq \mathbf{0}\right)$ [17][18][21][22].

In the prestress-stable configuration, the tension applied to the members stabilizes the structure by avoiding the formation of zero-stiffness modes.

Finally, it is sayd a superstable configuration the one that corresponds to a prestressable tensegrity structure and to a nonnegative value of $\boldsymbol{K}_{G}$. 
This means that a superstable structure is stable along all the possible virtual displacements from the analyzed configuration and it does not depend on prestress and material properties [17].

\section{FORM-FINDING OF CLASS THETA TENSEGRITY PRISMS}

This Section illustrates a mathematical approach employed to optimize the shape of a Class $\boldsymbol{\theta}=1$ tensegrity tetrahedron [24][25]. We first consider the tetrahedron $\mathbf{T}_{2}$ illustrated in Fig. 1. The analysed structure is formed by four bars of length $b$ and two sets of four strings of length $c$ (internal cables) and $l$ (external cables). On introducing the Cartesian frame $\{\mathrm{O}, \mathrm{x}, \mathrm{y}, \mathrm{z}\}$ depicted in red in Fig. 1, we can obtain the nodal coordinate vectors depending on $c, l$ and $\alpha$, that denotes the arbitrary angle between internal strings and the y-axis.
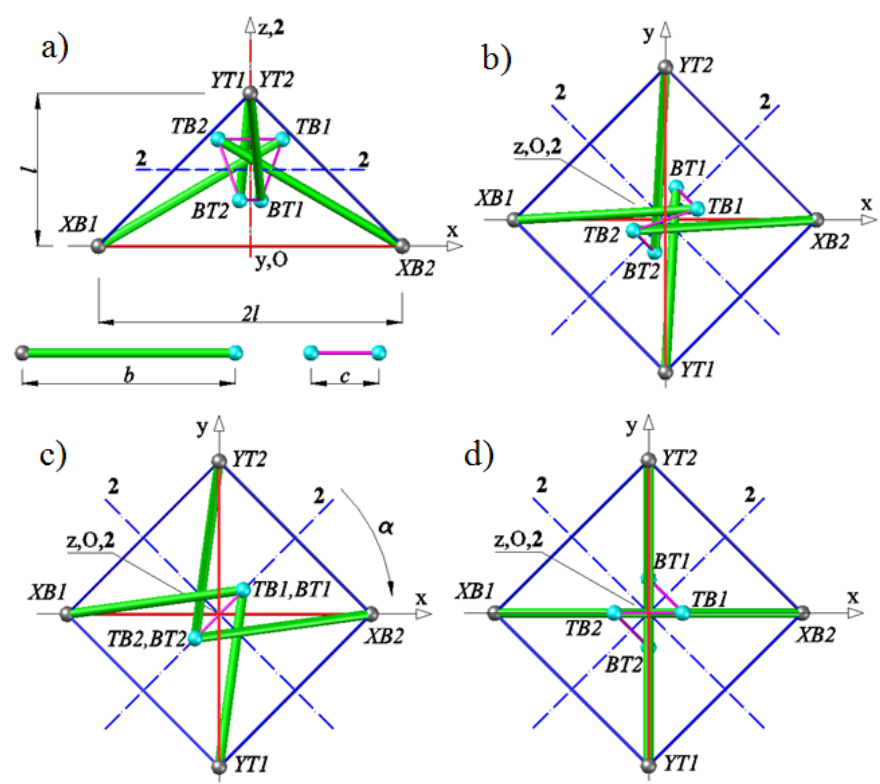

Figure 1: Class $\theta=1$ tensegrity tetrahedron: a) front view of the reference configuration, b) top view of the reference configuration, c) top view of model in the first extreme position for $\alpha=0^{\circ}, d$ ) top view of model in the second extreme position for $\alpha=45^{\circ}$ [25].

The length of the bars can be easily computed by using the following espression:

$$
b=\left[\frac{1}{4} c^{2}+l^{2}+\frac{1}{4}(l+c \cos \alpha)^{2}+l c \cos \left(\frac{\pi}{4}-\alpha\right)\right]^{\frac{1}{2}}
$$

The geometrically feasible configurations of the tensegrity tetrahedron are obtained by letting $\alpha$ vary between $\alpha=0^{\circ}$ (Figure $1 \mathrm{c}$ ) and $\alpha=45^{\circ}$ (Figure $1 \mathrm{~d}$ ).

We now consider the class $\theta=1$ triangular tensegrity prism centered at the origin of a Cartesian frame $\{\mathrm{O}, \mathrm{x}, \mathrm{y}, \mathrm{z}\}[26]$ (Figure 3). 

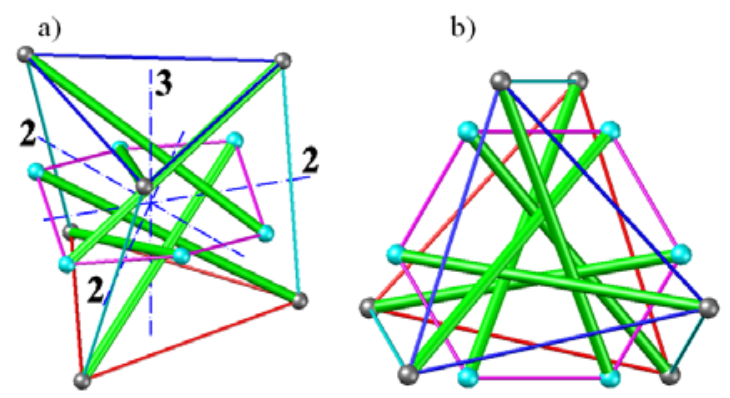

Figure 2: Class $\boldsymbol{\theta}=1$ triangular tensegrity prism.

This structure is formed by six bars of equal length $b$ and two sets of internal and external cables. Inner cables are formed by 6 elements of equal length $c$, connecting the nodes TB1BT1-TB2-BT2-TB3-BT3 (Figure 3).

The nine external cables include:

- $\quad$ two set of three horizontal cables with length $l$, connecting the nodes T1,T2,T3 and B1,B2,B3 (Fig.Figure 3);

- $\quad$ three horizontal cables T1-B1, T2-B2 and T3-B3 with equal length $v$ (Fig.Figure 3).

The kinematics of such a structure can be described by varying two aspect angles $\alpha$ and $\beta$ describing the slope of the internal strings with respect to the xy-axes and the twisting angle between the two external bases of the structure, respectively.

By varying the values of $\alpha$ and $\beta$ and the lengths of the connectivities, we could design different configurations. Fig.Figure 3 shows the extreme non-equilibrium configurations of the analysed structure for $\beta=0^{\circ}$ and $\beta=60^{\circ}$.

Few examples of quilibrium configurations are illustrated in Figure 4. In particular, Figure 4a corresponds to $l=v=2 c$ (regular configuration) and Figure $4 \mathrm{~b}$ corresponds to $l=v=c$ (expanded configuration).
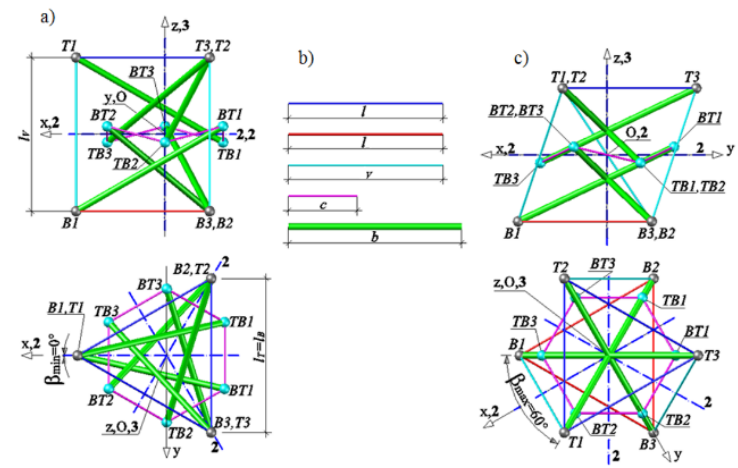

Figure 3: Front and top view of the extreme non-equilibrium configurations of the Class $\theta=1$ triangular tensegrity prism: a) tensegrity unit with $\beta \min =0^{\circ}$, b) elements forming the Class $\theta=1$ triangular tensegrity prism, c) tensegrity unit with $\beta \max =60^{\circ}$. 

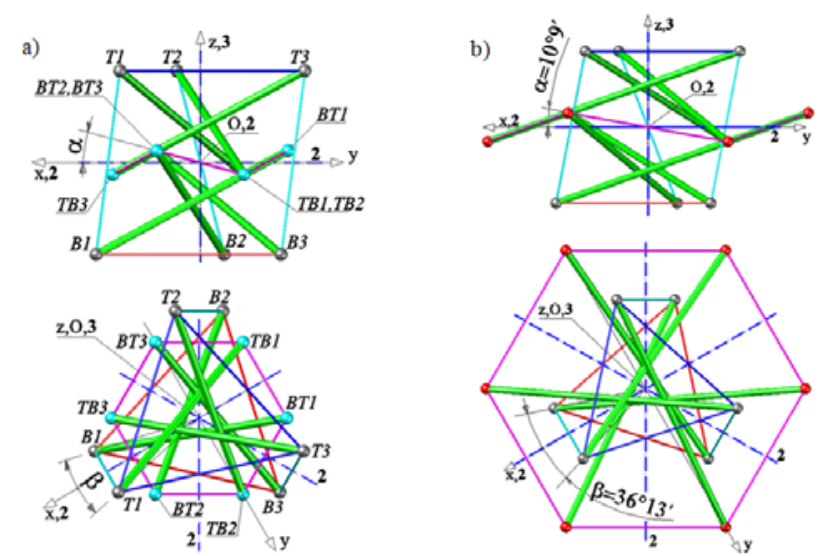

Figure 4: The self-stress equilibrium configurations of the unit with $l=v=2 c$ (a) and $l=v=c$ (b).

Making use of the previous results, we finally obtain [26]:

$$
\begin{gathered}
b=\left(\frac{1}{3}\left(l \cdot \cos \frac{\beta}{2}\right)^{2}+\frac{1}{4} c^{2}\left(1+3 \cos ^{2} \alpha\right)^{2}+c l \cdot \cos \alpha\left(\cos \frac{\beta}{2}+\frac{1}{3^{1 / 2}} \cdot \sin \frac{\beta}{2}\right)+\cdots\right. \\
\left.\ldots+\frac{1}{4} v^{2}+\frac{1}{2} c \cdot v\left(1-\frac{4}{3}\left(\frac{l}{v}\right)^{2} \cdot \sin ^{2}\left(\frac{\beta}{2}\right)\right)^{1 / 2} \cdot \sin \alpha\right)^{1 / 2}
\end{gathered}
$$

An approach to the form-finding of the illustrated tensegrity structures is to look for the zero this approach we refer to [16].

\section{PRESTRESS STABILITY AND SUPERSTABILITY}

Let us now study the stability problem of a tensegrity theta prism with reference to the model structure shown in Fig. 1. The freestanding configuration of the examined structure shows $l=$ $120 \mathrm{~mm}, v=125 \mathrm{~mm}$ and $c=55 \mathrm{~mm}$ [16]. The compressive members are made of grade 8.8, M4 threaded steel bars plated with white zinc, while the strings consist of Spectra ${ }^{\circledR}$ cables with $0.76 \mathrm{~mm}$ diameter [23].

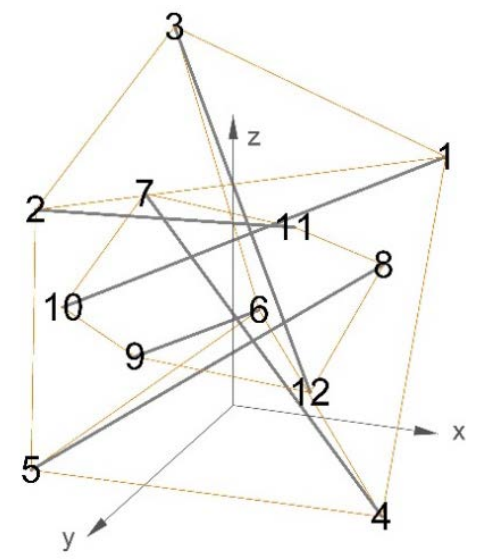

Figure 5: Freestanding configuration of a class theta tensegrity prism corresponding to $=120 \mathrm{~mm}, v=125 \mathrm{~mm}$ and $c=55 \mathrm{~mm}$. 
We begin by studying the kinematic problem of the structure in Fig. 1 through the approach presented in [16], which leads us to recognize that such a structure exhibit the ten infinitesimal mechanisms shown in Fig. 2 form the freestanding configuration (upon constraining rigid body motions).

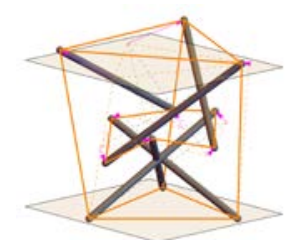

\#1

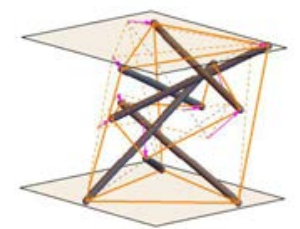

\#6

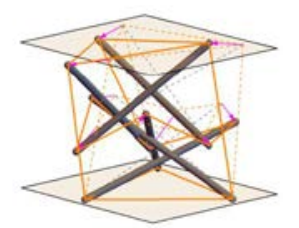

\#2

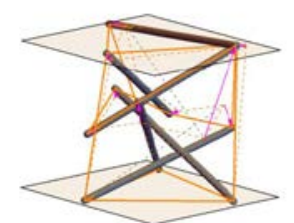

\#7

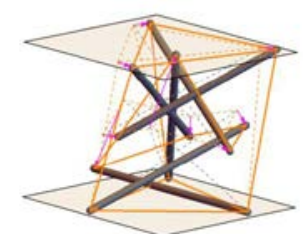

\#3

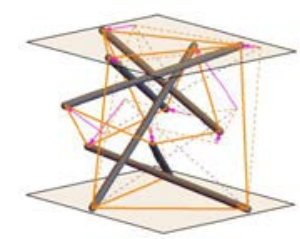

\#8

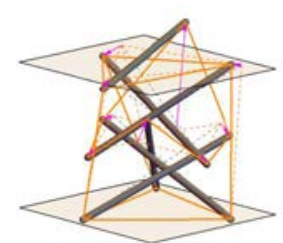

\#4

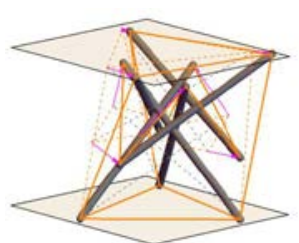

$\# 9$

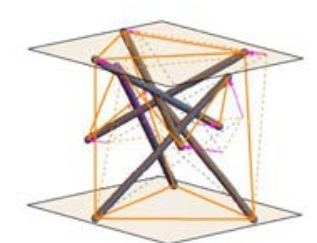

\#5

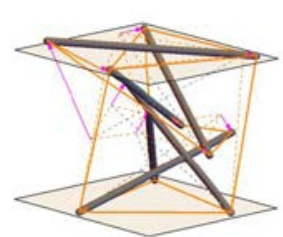

\#10

Figure 6: Infinitesimal mechanisms of the structure in Fig. Figure 5 from the freestanding configuration.

Next, we pass to compute the geometrical tangent stiffness matrix $\left(K_{T G}\right)$ of the structure under consideration employing the approach outlined in Sect. 2 and diffusely described in [16].

The self-states of self-stress in the freestanding configuration is characterized by the following force densities $x_{i-j}$ (positive in tension in the strings, and in compression in the bars), which are parametrized in terms of the force density $x$ acting in the base strings (free parameter). The force densities acting in the vertical strings are equal to $1.01 x$, while those acting in the internal strings are computed as follows: $x_{10-7}=x_{11-8}=x_{12-9}=3.61 x, x_{7-11}=x_{8-12}=x_{9-10}=$ $4.44 x$. Finally, the force densities acting in the bars are equal to $1.79 x$ in the self-stressed state. The geometric stiffness matrix of the structure, $K_{T G}$, can be cast into the following block form

$$
K_{T G}=x\left[\begin{array}{c:c:c:c}
G_{1} & G_{2} & 0 & G_{3} \\
\hdashline G_{2} & G_{4} & G_{5} & 0 \\
\hdashline 0 & G_{5} & G_{6} & G_{7} \\
\hdashline G_{3} & 0 & G_{8} & G_{9}
\end{array}\right]
$$

where the block-matrices $G_{i}(i=1, \ldots, 9)$ are given by:

$$
G_{1}=\left[\begin{array}{ccccccccc}
0.96 & 0.23 & 0.72 & -0.45 & 0.5 & 0 & -0.093 & -0.29 & 0 \\
0.23 & 0.77 & 0.032 & 0.5 & -0.55 & 0 & -0.29 & -0.91 & 0 \\
0.72 & 0.032 & 0.7 & 0 & 0 & -1 & 0 & 0 & -1 \\
-0.45 & 0.5 & 0 & 0.62 & -0.033 & -0.33 & -0.96 & -0.21 & 0 \\
0.5 & -0.55 & 0 & -0.033 & 1.1 & -0.64 & -0.21 & -0.045 & 0 \\
0 & 0 & -1 & -0.33 & -0.64 & 0.7 & 0 & 0 & -1 \\
-0.093 & -0.29 & 0 & -0.96 & -0.21 & 0 & 1 & -0.19 & -0.39 \\
-0.29 & -0.91 & 0 & -0.21 & -0.045 & 0 & -0.19 & 0.72 & 0.61 \\
0 & 0 & -1 & 0 & 0 & -1 & -0.39 & 0.61 & 0.7
\end{array}\right] \text {, }
$$




$$
\begin{aligned}
& G_{2}=\left[\begin{array}{ccccccccc}
-1 & 0 & 0 & 0 & 0 & 0 & 0 & 0 & 0 \\
0 & -0.95 & 0.23 & 0 & 0 & 0 & 0 & 0 & 0 \\
0 & 0.23 & -0.055 & 0 & 0 & 0 & 0 & 0 & 0 \\
0 & 0 & 0 & -0.97 & -0.024 & 0.2 & 0 & 0 & 0 \\
0 & 0 & 0 & -0.024 & -0.99 & -0.11 & 0 & 0 & 0 \\
0 & 0 & 0 & 0.2 & -0.11 & -0.055 & 0 & 0 & 0 \\
0 & 0 & 0 & 0 & 0 & 0 & -0.97 & 0.024 & -0.2 \\
0 & 0 & 0 & 0 & 0 & 0 & 0.024 & -0.99 & -0.11 \\
0 & 0 & 0 & 0 & 0 & 0 & -0.2 & -0.11 & -0.055
\end{array}\right] \text {, } \\
& G_{3}=\left[\begin{array}{ccccccccc}
0.59 & -0.43 & -0.72 & 0 & 0 & 0 & 0 & 0 & 0 \\
-0.43 & 1.6 & -0.26 & 0 & 0 & 0 & 0 & 0 & 0 \\
-0.72 & -0.26 & 1.4 & 0 & 0 & 0 & 0 & 0 & 0 \\
0 & 0 & 0 & 1.7 & -0.23 & 0.13 & 0 & 0 & 0 \\
0 & 0 & 0 & -0.23 & 0.48 & 0.76 & 0 & 0 & 0 \\
0 & 0 & 0 & 0.13 & 0.76 & 1.4 & 0 & 0 & 0 \\
0 & 0 & 0 & 0 & 0 & 0 & 1 . & 0.67 & 0.59 \\
0 & 0 & 0 & 0 & 0 & 0 & 0.67 & 1.2 & -0.49 \\
0 & 0 & 0 & 0 & 0 & 0 & 0.59 & -0.49 & 1.4
\end{array}\right] \text {, } \\
& G_{4}=\left[\begin{array}{ccccccccc}
0.96 & -0.23 & -0.72 & -0.093 & 0.29 & 0 & -0.45 & -0.5 & 0 \\
-0.23 & 0.77 & 0.032 & 0.29 & -0.91 & 0 & -0.5 & -0.55 & 0 \\
-0.72 & 0.032 & 0.7 & 0 & 0 & -1 & 0 & 0 & -1 \\
-0.093 & 0.29 & 0 & 1 . & 0.19 & 0.39 & -0.96 & 0.21 & 0 \\
0.29 & -0.91 & 0 & 0.19 & 0.72 & 0.61 & 0.21 & -0.045 & 0 \\
0 & 0 & -1 & 0.39 & 0.61 & 0.7 & 0 & 0 & -1 \\
-0.45 & -0.5 & 0 & -0.96 & 0.21 & 0 & 0.62 & 0.033 & 0.33 \\
-0.5 & -0.55 & 0 & 0.21 & -0.045 & 0 & 0.033 & 1.1 & -0.64 \\
0 & 0 & -1 & 0 & 0 & -1 & 0.33 & -0.64 & 0.7
\end{array}\right] \\
& G_{5}=\left[\begin{array}{ccccccccc}
0 & 0 & -1 & 0 & 0 & -1 & 0.33 & -0.64 & 0.7 \\
0.43 & 0.43 & 0.72 & 0 & 0 & 0 & 0 & 0 & 0 \\
0.72 & -0.26 & 1.4 & 0 & 0 & 0 & 0 & 0 & 0 \\
0 & 0 & 0 & 1 . & 0.67 & -0.59 & 0 & 0 & 0 \\
0 & 0 & 0 & -0.67 & 1.2 & -0.49 & 0 & 0 & 0 \\
0 & 0 & 0 & -0.59 & -0.49 & 1.4 & 0 & 0 & 0 \\
0 & 0 & 0 & 0 & 0 & 0 & 1.7 & 0.23 & -0.13 \\
0 & 0 & 0 & 0 & 0 & 0 & 0.23 & 0.48 & 0.76 \\
0 & 0 & 0 & 0 & 0 & 0 & -0.13 & 0.76 & 1.4
\end{array}\right] \text {, } \\
& G_{6}=\left[\begin{array}{ccccccccc}
4.4 & -2.2 & 0.3 & 0 & 0 & 0 & 0 & 0 & 0 \\
-2.2 & 2.1 & -0.11 & 0 & 0 & 0 & 0 & 0 & 0 \\
0.3 & -0.11 & 6.1 & 0 & 0 & 0 & 0 & 0 & 0 \\
0 & 0 & 0 & 4.6 & 2.1 & -0.24 & 0 & 0 & 0 \\
0 & 0 & 0 & 2.1 & 1.9 & -0.2 & 0 & 0 & 0 \\
0 & 0 & 0 & -0.24 & -0.2 & 6.1 & 0 & 0 & 0 \\
0 & 0 & 0 & 0 & 0 & 0 & 0.72 & 0.097 & -0.056 \\
0 & 0 & 0 & 0 & 0 & 0 & 0.097 & 5.7 & 0.31 \\
0 & 0 & 0 & 0 & 0 & 0 & -0.056 & 0.31 & 6.1
\end{array}\right] \text {, } \\
& G_{7}=\left[\begin{array}{ccccccccc}
-3.6 & 0 & 0 & -1.4 & 1.8 & -1 . & 0 & 0 & 0 \\
0 & -0.28 & 0.96 & 1.8 & -3.4 & -0.59 & 0 & 0 & 0 \\
0 & 0.96 & -3.3 & -1 . & -0.59 & -4.1 & 0 & 0 & 0 \\
0 & 0 & 0 & -1.1 & -1.4 & 0.83 & -4.4 & 0 & 0 \\
0 & 0 & 0 & -1.4 & -2.8 & -0.48 & 0 & -0.34 & 1.2 \\
0 & 0 & 0 & 0.83 & -0.48 & -3.3 & 0 & 1.2 & -4.1 \\
-1.4 & -1.8 & 1 . & 0 & 0 & 0 & -1.1 & 1.4 & -0.83 \\
-1.8 & -3.4 & -0.59 & 0 & 0 & 0 & 1.4 & -2.8 & -0.48 \\
1 . & -0.59 & -4.1 & 0 & 0 & 0 & -0.83 & -0.48 & -3.3
\end{array}\right] \text {, }
\end{aligned}
$$




$$
\begin{gathered}
G_{8}=\left[\begin{array}{ccccccccc}
-3.6 & 0 & 0 & 0 & 0 & 0 & -1.4 & -1.8 & 1 . \\
0 & -0.28 & 0.96 & 0 & 0 & 0 & -1.8 & -3.4 & -0.59 \\
0 & 0.96 & -3.3 & 0 & 0 & 0 & 1 . & -0.59 & -4.1 \\
-1.4 & 1.8 & -1 . & -1.1 & -1.4 & 0.83 & 0 & 0 & 0 \\
1.8 & -3.4 & -0.59 & -1.4 & -2.8 & -0.48 & 0 & 0 & 0 \\
-1 & -0.59 & -4.1 & 0.83 & -0.48 & -3.3 & 0 & 0 & 0 \\
0 & 0 & 0 & -4.4 & 0 & 0 & -1.1 & 1.4 & -0.83 \\
0 & 0 & 0 & 0 & -0.34 & 1.2 & 1.4 & -2.8 & -0.48 \\
0 & 0 & 0 & 0 & 1.2 & -4.1 & -0.83 & -0.48 & -3.3
\end{array}\right], \\
G_{9}=\left[\begin{array}{cccccccccc}
4.4 & 2.2 & -0.3 & 0 & 0 & 0 & 0 & 0 & 0 \\
2.2 & 2.1 & -0.11 & 0 & 0 & 0 & 0 & 0 & 0 \\
-0.3 & -0.11 & 6.1 & 0 & 0 & 0 & 0 & 0 & 0 \\
0 & 0 & 0 & 0.72 & -0.097 & 0.056 & 0 & 0 & 0 \\
0 & 0 & 0 & -0.097 & 5.7 & 0.31 & 0 & 0 & 0 \\
0 & 0 & 0 & 0.056 & 0.31 & 6.1 & 0 & 0 & 0 \\
0 & 0 & 0 & 0 & 0 & 0 & 4.6 & -2.1 & 0.24 \\
0 & 0 & 0 & 0 & 0 & 0 & -2.1 & 1.9 & -0.2 \\
0 & 0 & 0 & 0 & 0 & 0 & 0.24 & -0.2 & 6.1
\end{array}\right] .
\end{gathered}
$$

By prescribing a prestrain $p=0.1 \%$ in the base strings, we get $x=113.41 \mathrm{~N} / \mathrm{m}$ Let us refer to this value of the free parameter to numerically characterize the self-stress state of the structure under examination. It is easily verified that such a structure is prestress-stable since the work done by KG for each of the mechanisms in Figure 6 is positive. In fact, it results:

$$
\begin{aligned}
& K_{G} \cdot \hat{u}_{1}=324.56, K_{G} \cdot \hat{u}_{2}=224.581, K_{G} \cdot \hat{u}_{3}=596.017, K_{G} \cdot \hat{u}_{4}=711.805, \\
& K_{G} \cdot \hat{u}_{5}=345.87, K_{G} \cdot \hat{u}_{6}=400.564, K_{G} \cdot \hat{u}_{7}=739.289, K_{G} \cdot \hat{u}_{8}=506.349, \\
& K_{G} \cdot \hat{u}_{9}=711.595, K_{G} \cdot \hat{u}_{10}=449.881
\end{aligned}
$$

Making use of the Eigenvalues and Eigenvectors functions of Mathematica ${ }^{\circledR}$, we easily recognize that the KG matrix given by Eqn. (13) shows seven negative eigenvalues, which are associated with the eigenmodes graphically illustrated in Figure 7. We therefore conclude that such a structure is not superstable [17].
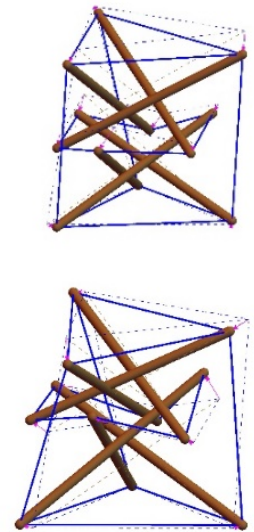

$\# 4$

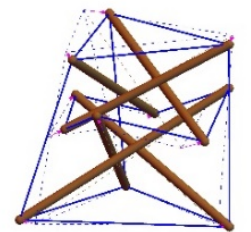

\#2

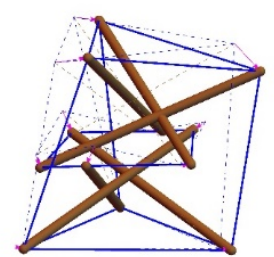

$\# 5$

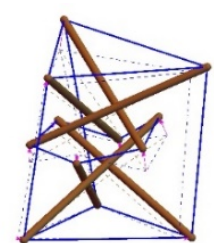

\#3

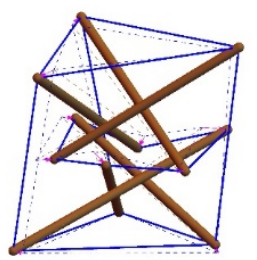

\#6

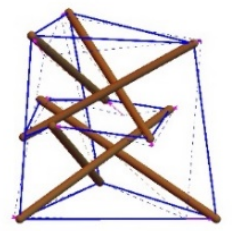

\#7

Figure 7: Eigenmodes associated with the negative eigenvalues of the geometric stiffness matrix. 


\section{CONCLUDING REMARKS}

We have reviewed the form-finding problem of class theta tensegrity prisms and have studied the stability problem of such structures with reference to an illustrative example. The given results have shown that the examined structure is prestress-stable but not superstable. This implies that it is not stable independently of the selfstress level and material properties [17]. The large number of infinitesimal mechanisms exhibited by tensegrity theta prisms justify the technical interest toward such structures, which are able to exhibit both soft and stiff modes [16]-[26]. Their use for the design of novel seismic metamaterials [27]-[28] and bandgap structures [29]-[31] is addressed to future work. We also plan to generalize the constitutive response of cables and bars to account for hyperelasticity, nonlinear, nonlocal and unilateral response of the material [32]-[45], through future research. Finally, we plan to design shells and membranes with tensegrity architecture [46]-[48] and to fabricate physical models of tensegrity structures through advanced additive manufacturing techniques [49]-[51].

\section{ACKNOWLEDGMENTS}

ZB gratefully acknowledges financial support from U-840/DS./H contract for statutory activities at Faculty of Civil and Environmental Engineering and Architecture at Rzeszów University of Technology. AA and FF gratefully acknowledge financial support from the Italian Ministry of Education, University and Research (MIUR) under the "Departments of Excellence"grant L.232/2016.

\section{REFERENCES}

[1] R. E. Skelton, M. C. de Oliveira, Tensegrity Systems, Springer, 2010c.

[2] F. Fraternali, L. Senatore, C. Daraio, Solitary waves on tensegrity lattices. J.Mech. Phys. Solids, 60, 1137-1144, 2012.

[3] F. Fraternali, G. Carpentieri, A. Amendola, On the mechanical modeling of the extreme softening/stiffening response of axially loaded tensegrity prisms. J. Mech. Phys. Solids, 74, 136-157, 2014.

[4] A. Amendola, G. Carpentieri, M. De Oliveira, R.E. Skelton, F. Fraternali, Experimental investigation of the softening-stiffening response of tensegrity prisms under compressive loading. Compos. Struct., 117, 234-243, 2014.

[5] F. Fraternali, G. Carpentieri, A. Amendola, R.E. Skelton, V. F. Nesterenko, Multiscale tunability of solitary wave dynamics in tensegrity metamaterials. Appl. Phys. Lett., 105, 201903, 2014.

[6] A. Amendola, E.H. Nava, R. Goodall, I. Todd, R.E. Skelton, F. Fraternali, On the additive manufacturing and testing of tensegrity structures. Compos. Struct., 131, 66-71, 2015.

[7] A.G. Tilbert, S. Pellegrino, Review of form-finding methods for tensegrity structures. Int. J. Space Struct., 18, 209-223, 2011.

[8] R.E. Skelton, Structural systems: a marriage of structural engineering and system science. J. Struct. Control, 9,113-133, 2002.

[9] R.E. Skelton, M. C. de Oliveira, Optimal complexity of deployable compressive structures. J. Franklin I., 347,228-256, 2010a. 
[10].E. Skelton, de Oliveira, M. C., 2010b. Optimal tensegrity structures in bending: the discrete Michell truss. J. Franklin I., 347, 257-283.

[11] R.E. Skelton, K. Nagase, Tensile tensegrity structures. Int. J. Space Struct., 27,131-137, 2012.

[12] K. Nagase, R.E. Skelton, Double-helix tensegrity structures. AIAA J., 2014. DOI: 10.2514/1.J053264.

[13] R. E. Skelton, F. Fraternali, G. Carpentieri, A. Micheletti, Minimum mass design of tensegrity bridges with parametric architecture and multiscale complexity. Mech Res. Commun., 58, 124-132, 2014.

[14] G. Carpentieri, R.E. Skelton, F. Fraternali, Minimum mass and optimal complexity of planar tensegrity bridges. Int. J. Space Struct., 30(3-4), 221-244, 2015.

[15] G. Carpentieri, R.E. Skelton, F. Fraternali, A minimal mass deployable structure for solar energy harvesting on water canals. Struct Multidiscip. Opt., 55(2), 449-458, 2017.

[16] M. Modano, I. Mascolo, F. Fraternali, Z. Bieniek, Numerical and analytical approaches to the self-equilibrium problem of class $\theta=1$ tensegrity metamaterials. Frontiers in Materials (Mechanics of Materials), 5:5, 2018.

[17] A. Micheletti, Bistable regimes in an elastic tensegrity system. Proc. R. Soc. A, 469:20130052, 2013.

[18] S.D., Guest, The stiffness of prestressed frameworks: a unifying approach. Int. J. Solids Struct., 43, 842-854, 2006.

[19] F. Fraternali, E. De Chiara, R.E. Skelton, On the use of morphing and wind stable tensegrity structures for shading facades of smart buildings. Smart Materials and Structures, 24, 105032 (10PP), 2015.

[20] R.E. Skelton, M.C. de Oliveira, Tensegrity Systems. Berlin: Springer, 2010.

[21] M. Schenk, S.D. Guest, J.L. Herder, Zero stiffness tensegrity structures, Int. J. Solids Struct., 44, 6569-6583, 2007.

[22] A . Favata, A. Micheletti, P. Podio-Guidugli, A nonlinear theory of prestressed elastic stick-and-strings structures. Int. J. Eng. Sci., 80, 4-20, 2014 .

[23] I. Mascolo, A. Amendola, G. Zuccaro, L. Feo, F. Fraternali, On the geometrically nonlinear elastic response of class $\theta=1$ tensegrity metamaterials. Frontiers in materials (Mechanics of materials), 5:16, 2018.

[24] Z. Bieniek, A mathematical model of the Class Theta tetrahedral tensegrity module, Lightweight Structures in Civil Engineering - Contemporary Problems, XXI LSCE, Local Seminar of IASS Polish Chapter, editors: Romuald Tarczewski and Zbigniew Bieniek: Rzeszów University of Technology, 9-16, 4 December 2015.

[25] Z. Bieniek, The self-equilibrium problem of the Class-Theta tetrahedral tensegrity module, Composites Part B, 115, 21-29, 2017.

[26] Bieniek Z., The self-equilibrium configurations for the Class-Theta triangular tensegrity prism, Proceedings of the XXIII Conference AIMETA 2017, Salerno, Italy, 4-7 September 2017

[27] F. Fraternali, A. Amendola, Mechanical modeling of innovative metamaterials alternating pentamode lattices and confinement plates. J Mech Phys Solids, 99, 259-271, 2017.

[28] A. Amendola, G. Benzoni, F. Fraternali, Non-linear elastic response of layered structures, alternating pentamode lattices and confinement plates. Compos Part B-Eng, 115, 117-123, 2017. 
[29] A. Amendola, A. Krushynska, C. Daraio, N. M. Pugno, F. Fraternali, Tuning frequency band gaps of tensegrity mass-spring chains with local and global prestress. Int $J$ Solids Struct, 155, 47-56, 2018.

[30] Mancusi, G., Fabbrocino, F., Feo, L., Fraternali, F., Size effect and dynamic properties of 2D lattice materials, Composites Part B: Engineering, 112, 235-242, 2017.

[31] A. O Krushynska, A. Amendola, F. Bosia, C. Daraio, N. M. Pugno, F. Fraternali, Accordion-like metamaterials with tunable ultra-wide low-frequency band gaps, New $J$ Phys, 20(7), 2018.

[32] R. Luciano, E. J. Barbero, Analytical expressions for the relaxation moduli of linear viscoelastic composites with periodic microstructure. Journal of Applied Mechanics, 62(3), 1995.

[33] R. Luciano, J.R. Willis, Nonlocal constitutive response of a random laminate subjected to configuration-dependent body force. Journal of the Mechanics and Physics of Solids, 49(2), 431-444, 2001.

[34] T. Elsayed, A. Mota, F. Fraternali, M. Ortiz, A variational constitutive model for soft biological tissues. Journal of Biomechanics, 41(7), 1458-1466, 2008.

[35] I. Simonini, M. Angelillo, A. Pandolfi, Theoretical and numerical analysis of the corneal air puff test. Journal of the Mechanics and Physics of Solids, 93, 118-134, 2016.

[36] T. El Sayed, W. Mock, A. Mota, F. Fraternali, M. Ortiz. Computational Assessment of Ballistic Impact on a High Strength Structural Steel/Polyurea Composite Plate. Computational Mechanics, 43(4), 525-534, 2009.

[37] L. Ascione, V.P. Berardi, A. Giordano, A., Spadea, S., Pre-buckling imperfection sensitivity of pultruded FRP profiles. Composites Part B: Engineering, 72, 206-212. 2015.

[38] F. Fraternali, S. Spadea, L. Ascione, Buckling behavior of curved composite beams with different elastic response in tension and compression. Composite Structures, 100, 280-289, 2013.

[39] M. Angelillo, Static analysis of a Guastavino helical stair as a layered masonry shell. Composite Structures, 119, 298-304, 2015.

[40] A. Gesualdo, C. Cennamo, A. Fortunato, G. Frunzio, M. Monaco, A. Angelillo, Equilibrium formulation of masonry helical stairs, Meccanica, 52 (8), 1963-1974, 2017.

[41] M. Angelillo, E. Babilio, A.Fortunato, M. Lippiello, A. Montanino, Analytic solutions for the stress field in static sandpiles. Mechanics of Materials, 95, 192-203, 2016.

[42] R. Luciano, J.R. Willis, Boundary-layer corrections for stress and strain fields in randomly heterogeneous materials. Journal of the Mechanics and Physics of Solids, 1075-1088, 2003.

[43] R. Luciano, J.R. Willis, FE analysis of stress and strain fields in finite random composite bodies. Journal of the Mechanics and Physics of Solids, 53(7), 505-1522, 2005.

[44] R. Luciano, J.R. Willis, Hashin-Shtrikman based FE analysis of the elastic behaviour of finite random composite bodies. International Journal of Fracture, 137(1), 261-273, 2006.

[45] A. Fortunato, F. Fraternali, A. Angelillo, Structural capacity of masonry walls under horizontal loads. Ingegneria Sismica/International Journal of Earthquake Engineering, 31(1), 41-51, 2014. 
[46] F. Fraternali, J.N. Reddy, A Penalty Model for the Analysis of Laminated Composite Shells. International Journal of Solids and Structures, 30, 3337-3355, 1993.

[47] B. Schmidt, F. Fraternali, Universal formulae for the limiting elastic energy of membrane networks. Journal of the Mechanics and Physics of Solids, 60, 172-180, 2012.

[48] F. Fraternali, G. Carpentieri, M. Modano, F. Fabbrocino, R.E. Skelton, A tensegrity approach to the optimal reinforcement of masonry domes and vaults through fiberreinforced composite materials. Composite Structures, 134, 247-254, 2015.

[49] I. Farina, F. Fabbrocino, F. Colangelo, L. Feo, F. Fraternali, Surface roughness effects on the reinforcement of cement mortars through 3D printed metallic fibers. Composites. Part B, Engineering, 99, 305-311, 2016.

[50] R. Singh, R. Kumar, L. Feo, F. Fraternali, Friction welding of dissimilar plastic/polymer materials with metal powder reinforcement. Composites. Part B, Engineering, 101, 77-86, 2016.

[51] D. Han, Z. Lu, S. A. Chester, H. Lee, Micro 3D printing of a temperature-responsive hydrogel using projection micro-stereolithography. Science Report 8(1):1936, 2018. 